\title{
APPLICATION OF SYNCHROTRON RADIATION TO THE ATOMIC AND ELECTRONIC STRUCTURE OF SEMICONDUCTORS
}

\author{
M. Altarelli
}

European Synchrotron Radiation Facility, BP 220, 38043 Grenoble Cedex, France

\begin{abstract}
A brief review of the main experimental techniques exploiting synchrotron radiation in semiconductor physics is attempted. Topics emphasized include the study of surface and interface phenomena, such as surface structural properties (e.g. surface reconstruction) by X-ray diffraction, surface dynamical properties (e.g. adsorbate vibrational amplitudes) by the $\mathrm{X}$-ray standing waves technique, etc. This review emphasizes brilliance (the phase-space density of photons) as the main figure of merit for many experimental techniques applicable to research in semiconductor physics. Examples of experiments made possible by the so-called "third generation", high-brilliance synchrotron sources are presented.
\end{abstract}

PACS numbers: 68.35.Bs, 68.55.-a, 78.70.Ck

\section{Introduction}

In the last few decades, synchrotron radiation has produced a revolution in all experimental techniques using photons in the wavelength region from the vacuum ultraviolet to the hard X-rays. In this article, some of the applications of synchrotron radiation to semiconductor physics will be discussed. Emphasis is given to fundamental physical aspects, rather than to more practical applications to material sciences. Even then, space will not allow to describe all techniques of interest, so that some hard choices are to be made. Thus, for example, we shall not discuss photoemission spectroscopy, although it has been a tool of great importance for the investigation of bulk and surface electronic structure [1]. The choice of topics reflects the personal interests of the author and also the novel perspectives opened up by the new generation of synchrotron radiation sources, such as the European Synchrotron Radiation Facility, which recently started to operate in Grenoble. Therefore, some examples of application of surface X-ray diffraction to the study of semiconductor surfaces and interfaces will be discussed, followed by a description of a powerful technique, X-ray standing waves, which is almost exclusively applicable to semiconductor crystals, and provides an accurate 
determination of the average position as well as of the vibrational amplitudes for atomic layers near the surface. The consideration of these experimental techniques will lead us quite naturally to appreciate the role of brilliance of an X-ray source as the main figure of merit for many experiments. At this point the possibilities opened up by the new generation of synchrotron radiation sources, which are characterized by an increase in brilliance of at least two or three orders of magnitudes, will be discussed. The example of coherent scattering of X-rays will allow us to appreciate the new horizons opened up by this recent generation of machines. Our aim is therefore to stimulate the interest of the reader on the possibilities offered by synchrotron based techniques for semiconductor physics, with no pretension to provide a satisfactory coverage of such an extensive field. The interested reader should turn to the excellent in-depth reviews of all aspects of synchrotron radiation and its applications which are to be found in Refs. $[2,3]$.

\section{Surface X-ray diffraction}

In recent years, an increasing interest in the use of X-rays for the study of surfaces and interfaces has developed [4,5]. When compared with electron diffraction techniques such as LEED (low-energy electron diffraction) and RIIEED (reflection high-energy electron diffraction), the main advantage of X-rays is the much smaller cross-section of interaction with atoms (of order $\left(Z r_{0}\right)^{2}$, where $Z$ is the atomic number, and $r_{0}=e^{2} / m c^{2} \approx 2.82 \times 10^{-13} \mathrm{~cm}$ is the classical electron radius; therefore, smaller than the corresponding electron-atom scattering cross-section by a factor $\sim(v / c)^{4}$, where $v$ is the electron velocity). This makes multiple scattering events, which dominate electron diffraction, very unlikely for $\mathrm{X}$-rays, so that the interpretation of the measured intensity profiles is much more straightforward. Furthermore, this makes the X-ray penetration depth very large (typically, up to several $\mu \mathrm{m}$ ), thus allowing the study of buried layers and interfaces. X-ray penetration also removes the constraint of an UHV environment, thus allowing, for instance, the in situ monitoring of CVD (chemical vapour deposition) growth, not accessible to electron-based techniques. A counterpart is that the small cross-section makes X-rays much less sensitive to surface atoms. This problem is circumvented by reducing the incidence angle below the critical angle for total reflection, which is given by [6]:

$$
\theta_{c}=\left(\lambda^{2} \rho r_{0} / \pi\right)^{1 / 2},
$$

where $\lambda$ is the wavelength and $\rho$ - the electron density of the material. Under such grazing incidence conditions, the penetration depth is reduced to $10-20$ monolayers.

The geometry of a grazing incidence experiment is shown in Fig. 1. The momentum transfer $q=k_{\text {out }}-k_{\text {in }}$ is the crucial quantity, with components $q_{\|}$and $q_{\perp}$, respectively parallel and perpendicular to the surface. If $q_{\|}=0$, we speak of specular reflection and a reflectometry experiment consists in recording the outgoing (reflected) intensity as a function of $q_{\perp}$. Below the critical angle, the reflectivity is of course unity, above the critical angle it is reduced in a fashion which reflects the shape of the electron density distribution in the direction normal to the interface. In the idealized case of a constant electron density terminating abruptly at 


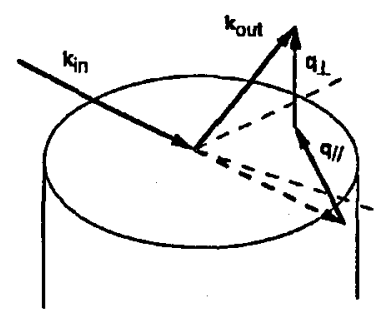

Fig. 1. Schematic illustration of the geometry of a surface diffraction experiment, showing the incoming and outgoing wave vectors, $k_{i n}$ and $k_{\text {out }}$, and the parallel and perpendicular components of the momentum transfer $q$.

the solid-vacuum interface, the reflected intensity falls off like $\left(q_{\perp} / q_{\mathrm{c}}\right)^{-4}$ (Fresnel reflectivity), where

$$
q_{c}=(4 \pi / \lambda) \sin \theta_{c} \approx 4\left(\rho r_{0} \pi\right)^{1 / 2}
$$

is the momentum transfer at the critical incidence angle. In real experiments, deviations from the Fresnel profile can be directly related to the profile of the charge drop-off at the surface.

On the other hand, in a surface diffraction experiment, the scattered intensity is recorded as a function of both components of $q$. In order to understand the kind of information that such experiments can provide, it is important to remember that (in the Born approximation) the scattering cross-section, and therefore the intensity of the scattered X-rays is proportional to the absolute square of the Fourier transform of the charge density

$$
I_{\mathrm{sc}}(\boldsymbol{q}) \approx r_{0}^{2}\left|\int \mathrm{d}^{3} r \rho(\boldsymbol{r}) \mathrm{e}^{-\mathrm{i} \boldsymbol{q} \cdot \boldsymbol{r}}\right|^{2} .
$$

Therefore, if the system retains full periodicity in the two directions parallel to the surface, intensity will be detected only if $q_{\|}$coincides with one of the two-dimensional reciprocal lattice ectors. If the surface is just the termination of an otherwise unperturbed three-dimensional crystal, the two-dimensional reciprocal lattice is the one associated to the unperturbed symmetry. If however, as it is very often the case for clean semiconductor surfaces, a reconstruction takes place, with a new two-dimensional periodicity, additional reflections will be observed. In the perpendicular direction, on the other hand, the presence of the surface always implies the loss of translational symmetry; consequently, intensity is collected in principle for all values of $q_{\perp}$. To each two-dimensional reciprocal lattice vector there is therefore associated a diffraction "rod", called crystal truncation rod [7], scanned by the continuous $q_{\perp}$ values, and with strong intensity peaks at those $q_{\perp}$ values which correspond to the position of three-dimensional reciprocal lattice vectors (Bragg peaks) in the infinite crystal. The study of the intensity distribution along the crystal truncation rods, especially in the low-intensity region in between two bulk Bragg peaks, delivers information on the charge distribution perpendicular to the surface, and together with the distribution of rods in the two-dimensional reciprocal space it gives a full crystallographic picture of the system. Note that, like all diffraction-based techniques, it provides information on 
the long-range order properties, which is complementary to local probes such as STM (scanning tunnelling microscopy).

There is a vast literature on surface diffraction with synchrotron radiation. Just as an example, we refer the reader to recent studies of the Ge(001) $(2 \times 1)$ reconstruction [8], or of the epitaxial deposition of $\mathrm{Ge}$ on $\mathrm{Ge}(111)$ [9].

In order to appreciate the importance of intense synchrotron sources in surface diffraction experiments, one must realize that with incidence angles of the order of the critical angle, in the mrad range, the projected sample dimension normal to the incident beam is of the order of $10 \mu \mathrm{m}$ for a $1 \mathrm{~cm}$ sample. In addition, the accuracy in angular measurement necessary for an accurate determination of the transferred momenta puts an equally demanding requirement on the angular divergence of the incoming beam (typically of order $10^{-4} \mathrm{rad}$ ). It is therefore necessary to have a photon flux sufficiently high to extract the weakest part of the crystal truncation rod in a very small area and with high angular collimation.

\section{X-ray standing waves}

The dynamical theory of X-ray diffraction is only applicable to crystals which can be regarded as perfect over distances of the order of the X-ray extinction length, typically $1-10 \mu \mathrm{m}$, and in practice it is almost exclusively restricted to semiconductor crystals. Under Bragg reflection conditions there is a fixed phase relationship between the incoming X-ray wave and the diffracted one, which results in the formation of a standing wave with nodes and antinodes parallel to the reflecting crystal planes [10]. It is then possible to exploit the fact that, as the crystal is rocked in angle across the Bragg reflection, the relative phase between incoming and diffracted wave changes by $\pi$, so that at angles well below the Bragg reflection maximum the nodes of the electric field coincide with the atomic planes, while the antinodes (maxima) are located halfway between successive atomic planes; and, conversely, at angles well above the Bragg maximum, the antinodes coincide with the atomic positions [11]. Correspondingly, a large variation in the fluorescence or in the photoelectron yield is observed, these processes being strongly enhanced when the maxima of the electric field coincide with atomic positions [12] (see Fig. 2).

Consider the situation in which an adsorbate is present. As the incoming $\mathrm{X}$-ray beam is scanned in angle across the Bragg reflection, the fluorescence intensity shows a profile given by

$$
Y_{A}(\theta)=1+R_{G}(\theta)+2\left[R_{G}(\theta)\right]^{1 / 2} f_{\mathrm{c}, \boldsymbol{G}} \cos \left[\phi(\theta)-\Phi_{A}\right],
$$

where $Y_{A}$ denotes the fluorescence intensity of adsorbate atoms $A, 1$ is the normalized yield far from the Bragg reflection, corresponding to the incoming wave intensity, $R(\theta)$ is the intensity of the diffracted wave as a function of incidence angle, $f_{c, G}$ is the adsorbate "coherent fraction" (see below), $\phi(\theta)$ is the phase shift between incoming and diffracted waves, and $\Phi_{A}$ is an angle defining the position of the adsorbate plane with respect to the registry with the substrate atomic planes. The last term describes the effect of the interference producing the standing waves. Its amplitude contains a factor corresponding to the fraction of adsorbate atoms 

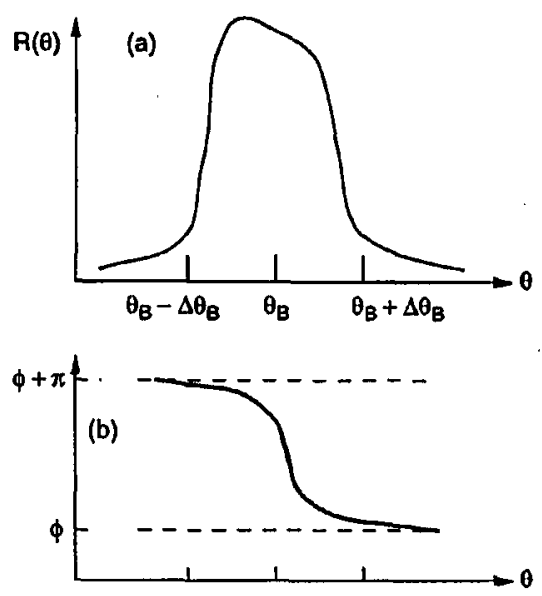

(c)

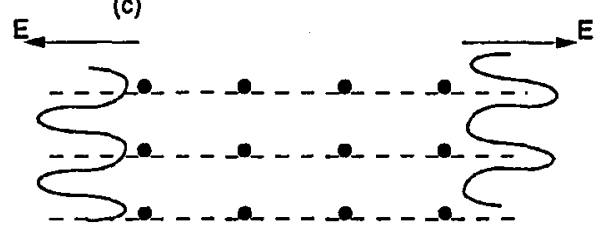

Fig. 2. (a) Reflectivity curve in the vicinity of the Bragg angle, $\theta_{\mathrm{B}}$. The characteristic width $\Delta \theta_{\mathrm{B}}$ is typically of the order of a few tens of $\mu \mathrm{rad}$; (b) behaviour of the phase shift between incident and diffracted wave near the Bragg reflection; (c) schematic behaviour of the electric field standing wave pattern with respect to the atomic planes, shown schematically by the rows of black circles: left, for $\theta \approx \theta_{\mathrm{B}}-\Delta \theta_{\mathrm{B}}$; right for $\theta \approx \theta_{\mathrm{B}}+\Delta \theta_{\mathrm{B}}$.

which are effectively sitting at the preferred site. It does contain in turn two contributions, $f_{\mathrm{c}, G}=C D_{G}$, where $C$ is the fraction of adsorbates which are sitting at the prescribed site and not in some random location; and $D_{G}$ is a temperature dependent Debye-Waller factor, which accounts for the weakening due to the thermal vibrations excursion of the adsorbate around its ideal position,

$$
D_{\boldsymbol{G}}=\exp \left(-\left\langle u_{\boldsymbol{G}}^{2}\right\rangle / d_{\boldsymbol{G}}^{2}\right) \text {, }
$$

in terms of the mean square vibration amplitude in the $\boldsymbol{G}$ direction, with respect to the spacing of the lattice planes corresponding to $\boldsymbol{G}$.

Typical examples of application of the standing wave method are the investigation of adsorbate positions with high accuracy (down to $0.03 \AA$, typically) as exemplified by the study of $\mathrm{Ge}$ on $\mathrm{Si}(001)$ [13], and also dynamical studies of vibration amplitudes as a function of temperature, via $\mathrm{Eq}$. (4), determining the harmonic vs. anharmonic behaviour of bond stretching and bending, as exemplified by a recent study of $\mathrm{Ga}$ as an adsorbate on various reconstructed phases of $\operatorname{Si}(111)[14]$.

The performance of an X-ray standing wave experiment requires accurate angular scanning across the Bragg reflection profile, the so-called Darwin-Prins curve. In perfect crystals, the angular width of this reflection is $\approx 10 \mu \mathrm{rad}$, so that 
an extreme angular precision is required to make scans with many points across such a small range. The corresponding stringent requirement on the divergence of the incident monochromatic beam can be reconciled with an acceptable count rate only if the beam emerging from the source has sufficiently high intensity and high collimation to start with.

\section{Experiments with high-brilliance $X$-ray sources}

In the course of the previous sections we pointed out the requirements of the various experiments. It transpires that the important figure of merit for many experiments is the source "brilliance" or "spectral brightness". This quantity is the phase space density of photons, expressed as the number of photons emitted in a certain bandwidth around the given frequency, in a unit time, by the unit area of the source, in a unit solid angle, and is commonly measured in photons $/ \mathrm{s} / \mathrm{mm}^{2} / \mathrm{mrad}^{2} / 0.1 \%$ bandwidth. The pursuit of higher brilliance has stimulated a tremendous progress in the physics of storage rings for synchrotron radiation. A new generation of synchrotron sources is becoming available, which are characterized by a reduction, with respect to machines of the previous generation, of one to two orders of magnitude in the phase space volume of the electron (or positron) beam, and by the extensive use of insertion devices (undulators and wigglers) as sources of radiation. In the first machine of the new generation to become available for experiments, the ESRF, undulators have achieved brilliances of the order of $10^{18}$ photons $/ \mathrm{s} / \mathrm{mm}^{2} / \mathrm{mrad}^{2} / 0.1 \% \mathrm{BW}$.

The achievement of such higher brilliances, typically 2 to 3 orders of magnitude higher than those so far available, will not only provide for a dramatic improvement in the experiments already performed with synchrotron radiation, but also open the way to new. experiments not yet attempted and which could turn out to be very important for semiconductor physics and for materials science in general.

A very interesting consequence of the small source size and of the high brilliance of modern synchrotron sources is the possibility to perform experiments exploiting the spatial coherence of the X-ray beams. As it is well known from classical optics, the fields emitted at wavelength $\lambda$ from a source of linear size $\rho$ are characterized by a coherence length $L$, when viewed at a distance $d$ from the source, given by

$$
L \approx \lambda d / \rho \text {. }
$$

This means that if the beam is intercepted by a pinhole of size comparable or smaller than $L$, the pinhole constitutes a spatially coherent source, in the sense that the radiation emerging from all its points has nearly the same phase. In order to exploit this property, of course, one must have a sufficient intensity going through the pinhole of size $L$, whence the interest for small $\rho$ and high brilliance. Under realistic ESRF conditions, the coherence length $L$ at $40 \mathrm{~m}$ from the source is of the order of $10 \mu \mathrm{m}$.

A typical signature of the spatial coherence of the beam is the observation of diffraction phenomena on geometrically simple objects: the pinhole itself, 
which reproduces the Fraunhofer diffraction pattern of circular apertures, or the Fresnel diffraction from the edge of a crystal intercepted by the beam. The first phenomenon was observed both on a wiggler at the National Synchrotron Light Source (NSLS) in Brookhaven, USA [15], and on an undulator at ESRF [16]; the second was observed at ESRF.

In pioneering experiments first performed in Brookhaven [15], and more recently and under much more favourable conditions at ESRF, "speckle" scattering of X-rays was detected [16]. Speckle scattering is a phenomenon well known from laser physics, in which intensity fluctuations are detected in the scattering of a coherent beam by a sample, as a result of the interference between the beam components scattered by different domains or by regions of the sample undergoing fluctuations.

Speckle scattering at ESRF was observed on various samples, including a GaAs-AlGaAs superlattice grown by MBE (molecular beam epitaxy). The detection of speckle patterns and of their time dependence is a way to investigate the size of domains or grains, the dynamics of their evolution, and more generally, the spacial and temporal scale of fluctuations in the sample. While this is done more easily with visible photons from lasers, the length scale of fluctuations accessible is related to the wavelength of the probe: in going from visible light to X-rays, it is hoped that it can be reduced from thousands of $\AA$ to a few $\AA$. The speckle scattering observations on the superlattice established that the dominant form of disorder is related to fluctuations in the periods and in stacking, while the in-plane structure appeared more ideally ordered.

\section{References}

[1] See e.g. N.V. Smith, F.J. Himpsel, in: Handbook of Synchrotron Radiation, Vol. 1a, Ed. E.E. Koch, North-Holland, Amsterdam 1983.

[2] Neutron and Synchrotron Radiation for Condensed Matter Studies, Vol. I and II, Eds. J. Baruchel, J.L. Hodeau, M.S. Lehmann, J.R. Regnard, C. Schlenker, Springer, Berlin 1993-94.

[3] Handbook of Synchrotron Radiation, Vol. 1-3, Eds. E.E. Koch, T. Sasaki, H. Winick, North-Holand, Amsterdam 1983-1991.

[4] I.K. Robinson, in: Handbook of Synchrotron Radiation, Vol. 3, Eds. G.S. Brown, D.E. Moncton, North-Holland, Amsterdam 1991, p. 221.

[5] M. Sauvage-Simkin, in: Neutron and Synchrotron Radiation for Condensed Matter Studies, Vol. II, Eds. J. Baruchel, J.L. Hodeau, M.S. Lehmann, J.R. Regnard, C. Schlenker, Springer, Berlin 1994, p. 51.

[6] See e.g. J. Als-Nielsen, in: Structure and Dynamics of Surfaces II, Eds. W. Schommers, P. von Blankenhagen, Springer, Berlin 1987, p. 181.

[7] S.R. Andrews, R.A. Cowley, J. Phys. C 18, 6247 (1985); I.K. Robinson, Phys. Rev. $B$ 33, 3830 (1986).

[8] R. Rossman, H.L. Meyerheim, V. Jahns, J. Wever, W. Moritz, D. Wolf, D. Dornisch, H. Schulz, Surf. Sci. 279, 199 (1992).

[9] R.G. van Silfhout, J.F. van der Veen, S. Ferrer, C. Norris, Surf. Sci. 264, 281 (1992).

[10] M. von Laue, Ann. Phys. (Germany) 23, 705 (1935). 
[11] B.W. Batterman, H. Cole, Rev. Mod. Phys. 36, 681 (1964).

[12] B.W. Batterman, Phys. Rev. 133, A759 (1964).

[13] E. Fontes, J.R. Patel, F. Comin, Phys. Rev. Lett. 70, 2790 (1993).

[14] R.E. Martinez, E. Fontes, G. Golovchenko, J.R. Patel, Phys. Rev. Lett. 69, 1061 (1992).

[15] M. Sutton, S.G.J. Mochrie, T. Greytak, S.E. Nagler, L.E. Berman, G.A. Held, G.B. Stephenson, Nature 352, 608 (1991).

[16] G. Grübel, J. Als-Nielsen, D. Abernathy, G. Vignaud, S. Brauer, G.B. Stephenson, S.G.J. Mochrie, M. Sutton, I.K. Robinson, R. Fleming, R. Pindak, S. Dierker, J.F. Legrand, ESRF Newsletter 20, 14 (1994) and to be published. 